\title{
APPLICATIONS OF FREE SPACE OPTICS FOR BROADBAND ACCESS
}

\author{
E. Leitgeb ${ }^{1}$, M. Gebhart ${ }^{1}$, U. Birnbacher ${ }^{2}$, S. Sheikh Muhammad ${ }^{1}$, Ch. \\ Chlestil ${ }^{1}$ \\ ${ }^{I}$ Institute of Broadband Communications (at the Department of Communications and Wave \\ Propagation, INW), TU Graz, Inffeldgasse 12,8010 Graz, Austria, leitgeb@inw.tugraz.at \\ ${ }^{2}$ Institute of Communication Networks and Satellite Communications, TU Graz, Inffeldgasse \\ 12,8010Graz,Austria, birnbacher@inw.tugraz.at
}

\begin{abstract}
Free Space Optics (FSO) is an excellent supplement to conventional radio links and fibre optics. It is a broadband wireless solution for the "Last Mile" connectivity gap throughout metropolitan networks.

At the Department of Communications and Wave Propagation several FSOsystems have been developed within the past 5 years. Thereby the main effort was laid on using LEDs instead of laser diodes. One system is based on a modular concept using available standard components, used for distances of about $300 \mathrm{~m}$ at a specific power margin of $25 \mathrm{~dB} / \mathrm{km}$.

Additionally the research group "OptiKom" investigates the reliability and availability for different network-architectures (ring, mesh and star).
\end{abstract}

\section{INTRODUCTION}

Today, many people need high data rates for connecting to the Internet or for other access-services. Therefor Free Space Optics (FSO) is a well suited technology. The high bandwidth of the backbone (fibre network) is also available for the end user. The workgroup for Optical Communication ("OptiKom") at the Department of Communications and Wave Propagation, Technical University Graz, has carried out research in the field of Free Space Optics (FSO) over a period of about ten years. The work includes the development of equipment for research purposes and 
the evaluation of commercially available FSO systems for the climate in Graz (Austria) in co-operation with industrial partners. The main projects in this field have been funded by Telekom Austria AG, InfraServ Gendorf and the Government of Styria.

At the Department of Communications and Wave Propagation FSO-links are investigated and developed (in research projects and also in diploma and $\mathrm{PhD}$ theses). Additionally, existing systems are evaluated in cooperation with distributors, telephone companies and providers and optical links) are estimated in regard to range, bandwidth, traffic and weather (calculation of link budget and margin). In the work of "OptiKom" possibilities are investigated to increase the channel capacity and the reliability and availability in Optical Free Space Links.

\section{FREE SPACE OPTICS-APPLICATIONS}

Optical Wireless is the broadband solution (high data rates without any cabling) for connecting end users to the backbone (Last-Mile-Access). This technology is an excellent supplement to conventional radio links and fibre optics [3].

Applications for short range ("Last Mile", max. $1 \mathrm{~km}$ ) can be used as quick installation of optical wireless links in urban areas (Point-to-Point / Point-toMultipoint Systems) or as Broadband links for railway, highway or river crossings. Additionally FSO-links between buildings of companies or institutions (protection of , wiretapping') and rapid replacement of broken cable-links can be well suited solutions. To install FSO-systems for mobile / nomadic use (f.e. seminars, meetings, events) or links between different locations of dislocated events or for disaster management are also possibilities for using optical wireless technology.

In the following section different architectures are described and compared in regard to their advantages.

\subsection{Optical Wireless in Ring Architecture}

In the following schematic (figure 1) a FSO-network in ring architecture is shown. The distances between the buildings are up to $500 \mathrm{~m}$. In the minimum configuration two Optical Receiver / Transmitter Units (OSE) are installed on the top of each building. In the event of a broken link (failure) between building 1 and building 2 (direct connection), the indirect link can be used. Instead, the information is sent in the other direction of the ring network (building 1, building 6 , building 5 , building 4 , building 3 , and building 2 ). Thereby, a partial security against failure can be achieved.

The installation of additional links increases the availability and the security against failure (redundant links). An Optical Repeater has to be used, if there is no line of sight between transmitter and receiver. In a field test four FSO-links for short ranges (up to $300 \mathrm{~m}$ ) have been connected serially at the Department of Communications and Wave Propagation. In this experiment six of the connected FSO-units (three pairs) operated as repeaters. 


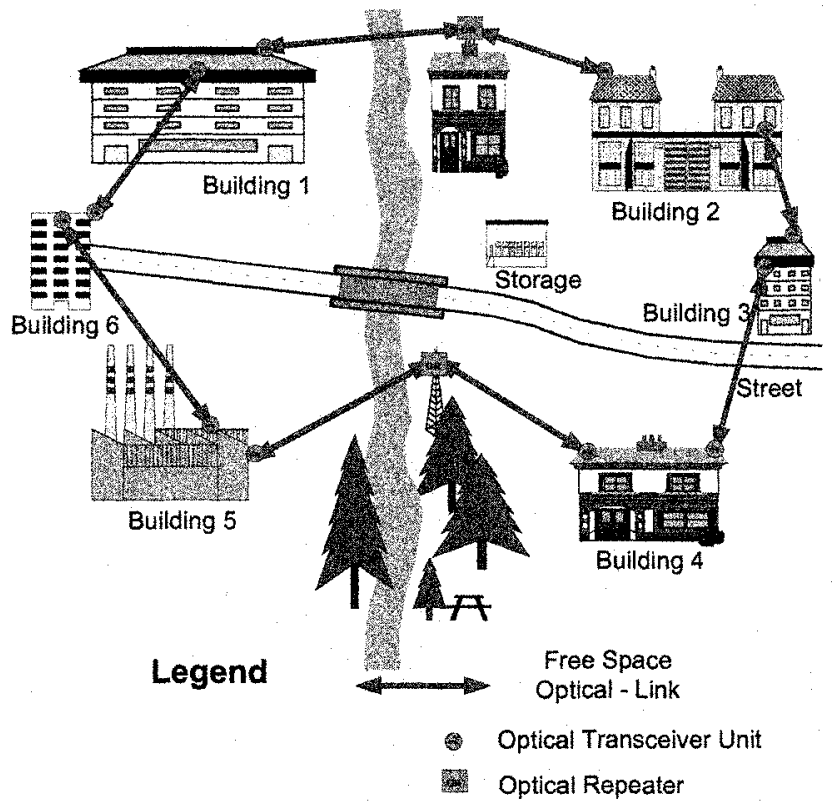

Figure 1. Optical Wireless (Ring Architecture)

\subsection{Optical Wireless in Star Architecture}

FSO networks with star architecture have been installed at the Department of Communications and Wave Propagation (project-group "OptiKom"). The coverage area of this FSO-network is about $300 \mathrm{~m}$ in diameter. An Optical Multipoint Unit (OZS) was mounted at the top of the department (Inffeldgasse 12). Five users (employees of the TU Graz) are permanently connected by their optical transceiver units to the Optical Multipoint hub-station. The five users are located inside the surrounding buildings (Inffeldgasse 10,12, 18 and 16). Thereby, the user PCs are connected via the FSO-link to the OZS. In this configuration the OZS is realised with five FSO-Point-to-Point units, each of them directed to user FSO terminal. The Optical Multipoint Unit is interconnected by switches with the backbone network of TU Graz.

The advantage of this configuration are the shorter distances between any two FSO-units, because the Optical Multipoint Unit (OZS) is used as a repeater. In general, the Optical Multipoint Unit is in the centre of the area, but this architecture has the disadvantage of a single point of failure. If the Optical Multipoint Unit fails, a system breakdown of the whole installation is caused. To improve the reliability of this architecture, a redundant Multipoint Unit has to be installed. A second Optical Multipoint can also be implemented on moveable platforms (e.g. a broadcast van). For the installation of Multipoint Units on cars (for short term use) FSO-systems with "autotracking" are preferable. A network in star architecture is 
shown in the following schematic (figure 2). The buildings are located in the same locations as in the previous map (section 2.1).

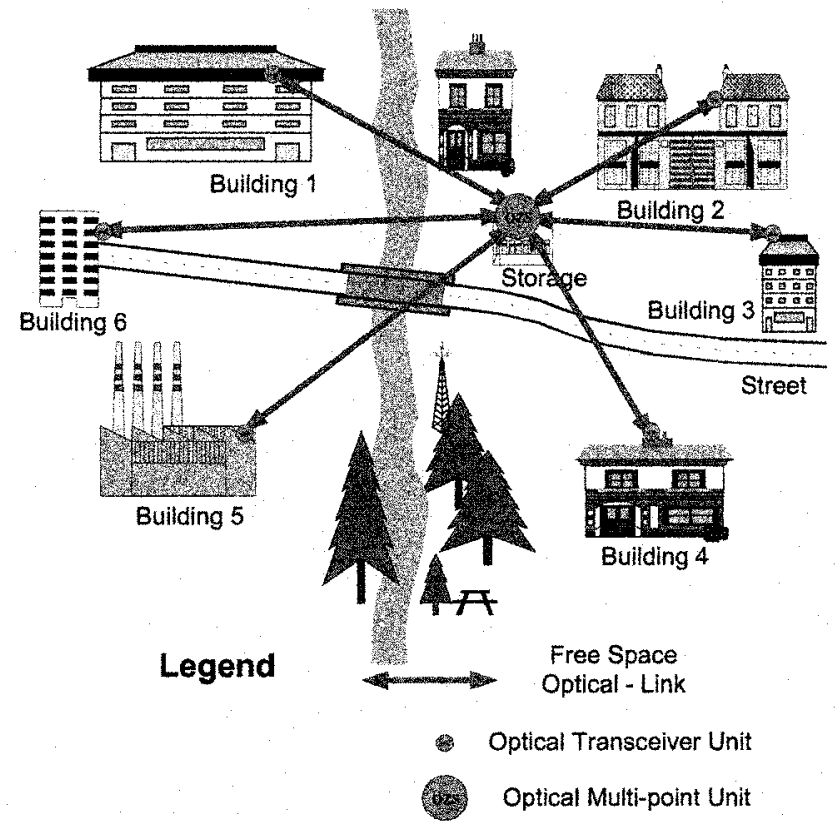

Figure 2. Optical Wireless (Star Architecture)

\subsection{Optical Wireless in meshed architecture}

For high reliability, the optimum network architecture is a meshed network, because it combines the advantages of the above described architectures. Different connections are possible, of which two examples (experimentally verified at the department) are described in this paper.

In figure 3 a mixture of a ring and a star network is shown. In each configuration, the central FSO-unit (OZS) can be connected to satellites, directional radio links, (mobile) telephone networks, or fibre optics. The Optical Multipoint Unit (OZS) can be connected with a switch or router to the backbone network. The clients in the buildings are linked with their FSO units to the OZS. This solution is similar to the FSO-network installed at the Department of Communications and Wave Propagation at the TU Graz.

Free Space Optics-systems with Light Emitting Diodes (LED) as source are developed [5] by the research-group "OptiKom" at the Department of Communications and Wave Propagation (section 3). Using special LEDs has the advantage that problems with Laser- and Eye Safety are minimised. The „OptiKom" group has developed low cost FSO systems for data rates of 10 and $100 \mathrm{Mbps}$ by using LEDs instead of laser diodes. Most of this work was funded by the government of Styria and InfraServ Gendorf. 


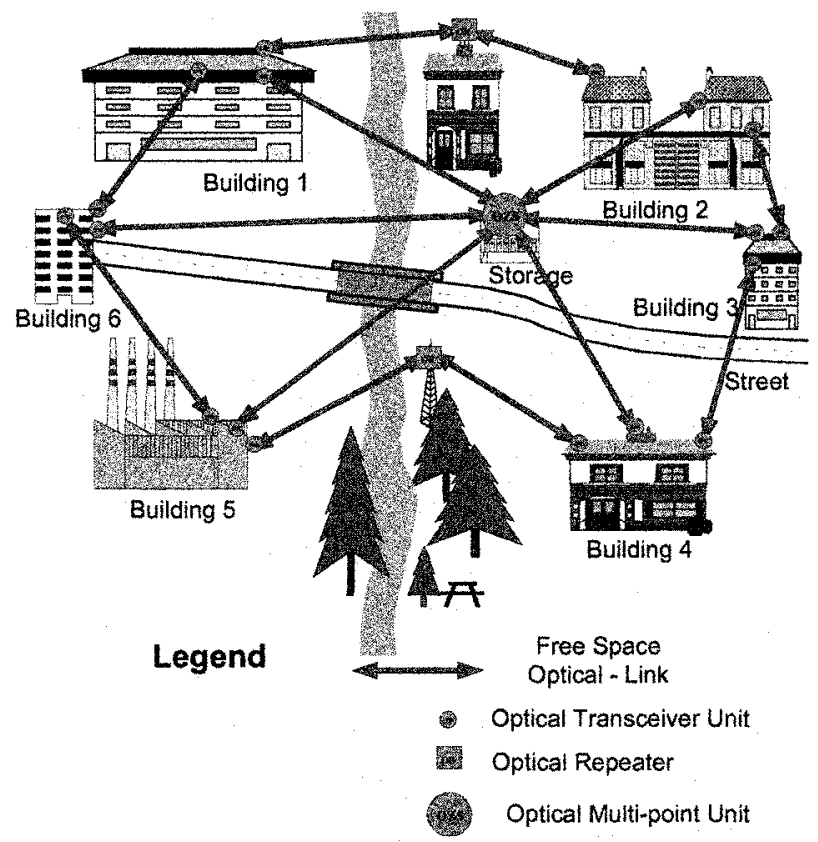

Figure 3. Optical Wireless (meshed architecture)

\section{DEVELOPED FSO-SYSTEMS WITH LIGHT EMITTING DIODES}

The developed systems combine available standard components to realise costeffective solutions. The beam divergence usually covers values from about 8 to 60 mrad, allowing an easy alignment for the user without the need of a telescope. All elements including the optics do not require a precision as high as needed for other approaches, allowing higher tolerances at production, and the use of simple mounts.

Due to large divergence, the requirements for a stable underground are not very high allowing a quick installation. Suitable distances for high availability operation are limited by the wide beam angle and depending on local climate to up to 300 meters. Due to Laser Safety Regulations the use of sources with larger emitting area allows more output power in the same safety class, which improves the link budget.

\subsection{Free Space Optics system for $100 \mathrm{~m}$}

The developed Free Space Optics system for a range of $100 \mathrm{~m}$ consists of two main parts, one transmitter (LED) and one receiver (photo-PIN-diode). The LED and the 
photo-PIN-diode are mounted in the focus of a cheap plastic lens encased by a tube of Aluminium alloy. The transmitter and receiver units are mounted on a printed circuit board in a housing of a video camera. On a single printed circuit board the electronic part of the Free Space Optics-system is located. The receiver electronic includes the photo-PIN-diode, the amplifier, and the data-interface. For the transmitter electronic, the data-interface, the driver amplifier and the LED are necessary. The beam divergence of this system is about two degrees. At the moment solutions are available for 10 and $100 \mathrm{Mbps}$.
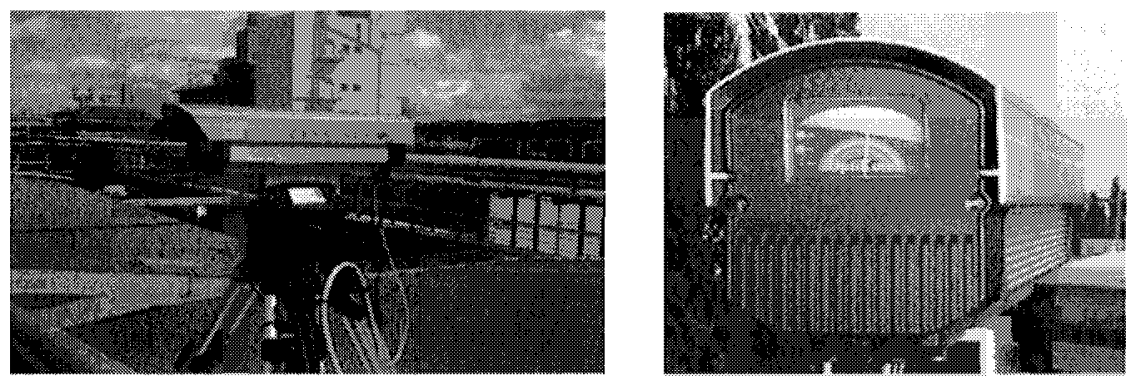

Figure 4. Developed Point-to-Point-system with LED (100 Mbps, $100 \mathrm{~m}$ ) A) Field-test in Germany (InfraServ Gendorf), B) Back view of the FSO-unit

The measured voltage of the receiver unit is shown on an analogue voltage display (integrated at the back of the system). The installation of this FSO-system is very easy, because of the beam divergence of two degrees and the above mentioned voltage display.

\subsection{Free Space Optics system for $\mathbf{3 0 0} \mathbf{~ m}$}

The developed Free Space Optics system for a range of $300 \mathrm{~m}$ is mounted in a plastic housing. The Free Space Optics-unit consists of 8 transmitters (LED) and one receiver (photo-PIN-diode). The LEDs are also mounted in the focus of a cheap plastic lens encased by a tube of Aluminium alloy. The received light is focused by a large lens to the photo-PIN-diode.
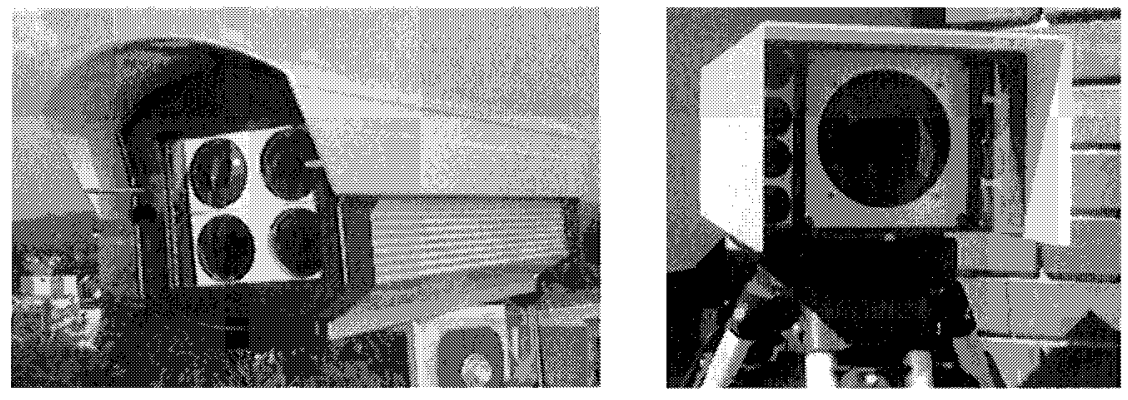

Figure 5. Developed Point-to-Point-System A) with 3 transmitter units (for $100 \mathrm{~m}$ ) and B) with 8 transmitter units (for $300 \mathrm{~m}$ ) 
The electronic part on the printed circuit board is similar to the small system for the $100 \mathrm{~m}$ range. By using VCSELs instead of LEDs, the range of the systems can be increased up to $800 \mathrm{~m}$.

\subsection{Folk-Festival "Aufsteirern" - Nomadic use of Free Space Optics}

A real service demonstration for nomadic use of optical wireless networks took place at the folk-festival "Aufsteirern 2002" in the historical town of Graz.

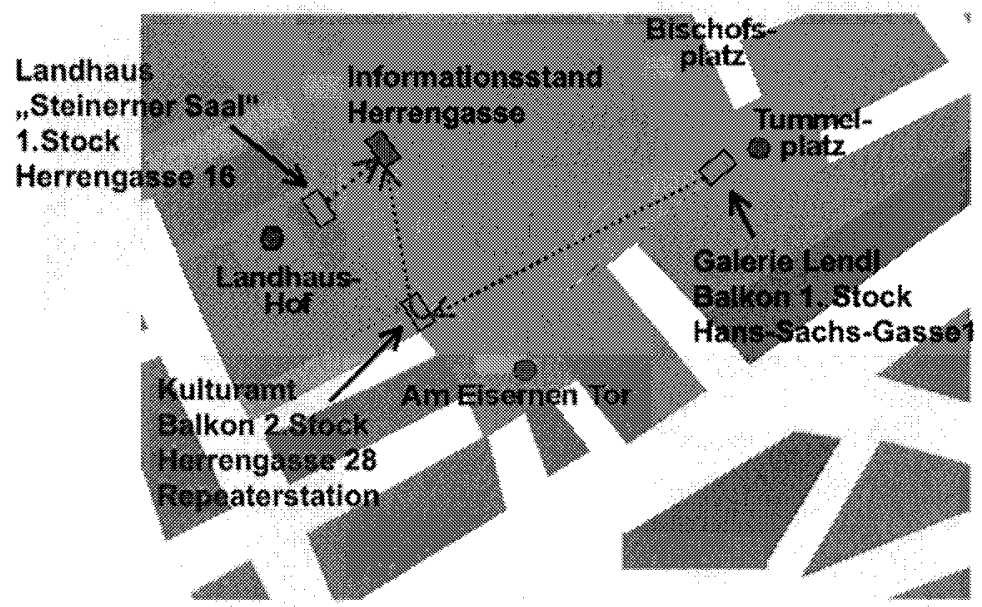

Figure 6. Location "Landhaushof" and „Tummelplatz" shown in the city map of Graz (Event ,Aufsteirern“)

Folk-dancers and brass bands were located at different places and streets. The task of the project-group "OptiKom" was to transmit live video pictures (with a data rate of $10 \mathrm{Mbps}$ ) from two locations ("Landhaushof" and "Tummelplatz") to the central enquiry kiosk ("Herrengasse"). Thereby, the visitors of the festival could inform themselves what was going on at the other locations "Landhaushof" and "Tummelplatz". In figure 6 the setup for this demonstration is shown in the city map of Graz. For the FSO links, the described systems were used to transmit live video data streams from the yard inside of an historical building ("Landhaushof") and from place ("Tummelplatz") to the enquiry kiosk ("Herrengasse"). The transmission from the location "Tummelplatz" was realised with the use of an optical repeater, because there was no line of sight between "Tummelplatz" and the enquiry kiosk. For this purpose two systems (for $300 \mathrm{~m}$ range) have been (inter)connected.

In a similar scenario we installed Free Space Optics- and Satellite applications at a civil-military exercise in Spring 2004 in Styria (southern part of Austria). In this civil-military cooperation we use a mobile Satellite Earth Station (equipped with 
Free Space Optics and Wireless LAN) for Videoconferencing between military and civil organisations (police, fire brigades and departments of the government). Additional Free Space Optics-links operate with high bit-rate for data-transfer between the military division and civil organisations (police etc.). This application is a new possibility of Broadband-Communication in Civil-Military-Cooperation (CIMIC).

\section{CONCLUSIONS}

Optical Wireless is an excellent nomadic broadband solution (high bandwidth) for connecting the end users to the backbone (Last-Mile-Access). This technology should be seen as supplement to conventional radio links and Fibre Optics. The use of low cost FSO-systems for short distances makes this technology interesting for private users.

At the moment the main work in this field is to increase reliability and availability. Those two parameters of the FSO-link are mainly determined by the local atmospheric conditions. So good reliability and availability can be achieved by using the Free Space Optics for short distances, by calculating enough link-budget and by using the optimal network architecture for each FSO application.

The optimal solution for FSO configurations is a meshed architecture. This network architecture combines shorter distances and high reliability, because of the location of the Optical Multipoint Unit (in the centre of the area).

The combination of FSO and microwave-links is also a further possibility for increasing reliability and availability, because terrestrial FSO is most effected by fog, whereas the microwave propagation is mainly influenced by rain [4]. Results of investigations show a reliability of $99.9991 \%$ for hybrid systems.

\section{REFERENCES}

[1] Leitgeb E., Bregenzer J., Fasser P., Gebhart M., Free Space Optics - Extension to Fibre-Networks for the "Last Mile", Proceedings and Presentation at IEEE / LEOS 2002 Annual Meeting, $10^{\text {th }}-14^{\text {th }}$ of November 2002, Glasgow, Scotland

[2] Leitgeb E., Gebhart M., Fasser P., Reliability of Free Space Laser Communications Investigations at the TU Graz, Proceedings. and Presentation at the $8^{\text {th }}$ Annual WCA Techn. Symp., $14^{\text {th }}-16^{\text {th }}$ of January 2002 , San Jose, CA, USA

[3] Leitgeb E., Gebhart M., Fasser P., Free Space Optical Access, Proceedings. and Presentation at tcmc2001, $15^{\text {th }} / 16^{\text {th }}$ of October. 2001, Graz

[4] Schrotter P., Birnbacher U., Kogler W., Leitgeb E., Koudelka O., Increased availability with hybrid optical / microwave networks, Presentation at WG1-Meeting COST 270, $9^{\text {th }} / 10^{\text {th }}$ of April 2002, Graz

[5] Gebhart M., Leitgeb E., Fasser P., Short-Range FSO-Systems - Increasing Network Flexibility and Reliability, Presentation at WG1-Meeting COST $270,9^{\text {th }} / 10^{\text {th }}$ of April 2002, Graz

[6] Leitgeb E., Gebhart M., Fasser P., Bregenzer J., Tanczos J., Impact of atmospheric effects in Free Space Optics transmission systems, Proceedings and Presentation at SPIE Photonics West, LASE 2003, $25^{\text {th }}-31^{\text {st }}$ of January 2003, San Jose, CA, USA 